\title{
ANÁLISE TEMPORAL DAS INTERNAÇÕES POR GRIPE E PNEUMONIA ASSOCIADAS ÀS VARIÁVEIS METEOROLÓGICAS NO MUNICÍPIO DE SÃO PAULO, SP
}

Marina Jorge de MIRANDA

\begin{abstract}
RESUMO
Este estudo investigou a associação dos elementos meteorológicos (umidade relativa mínima e temperaturas máxima e mínima diárias) com as internações por morbidades respiratórias (pneumonia e gripe), registradas por meio das notificações hospitalares para todas as faixas-etárias da população paulistana, utilizando técnica estatística de regressão linear. A incidência de internação diária de pessoas com problemas respiratórios do Sistema Único de Saúde (SUS), especificamente com influenza (gripe) e pneumonia, foi correlacionada com dados meteorológicos diários da estação meteorológica do Instituto de Astronomia e Geofísica da Universidade de São Paulo (IAG/USP) no período de janeiro de 2002 a dezembro de 2005. Pelos gráficos anuais de todo o período estudado, observamos uma certa tendência do aumento das internações quando há aumento das temperaturas máximas e mínimas, e queda da umidade relativa mínima em até 7 dias antes dos picos de internação. No entanto, os resultados dos testes de regressão linear indicaram uma baixa correlação entre o número de hospitalizações por influenza e pneumonia para quase todo o período. Apenas entre os meses de julho e setembro de 2004 que apresentaram correlações moderadas, tendo em vista que $47 \%$ das internações por influenza e pneumonia estavam associadas ao aumento das temperaturas mínimas e $35 \%$ das temperaturas máximas, coincidentes com os períodos mais secos do ano de 2004. Entre 2002 e 2005, destaca-se que os grupos de populações mais vulneráveis são as crianças de até 4 anos de idade e os idosos a partir de 60 anos.
\end{abstract}

Palavras-chave: Morbidade; Gripe; Pneumonia; Climatologia urbana; Município de São Paulo.

\section{ABSTRACT}

TEMPORAL ANALYSIS OF HOSPITAL ADMISSIONS FOR FLU AND PNEUMONIA ASSOCIATED WITH METEOROLOGICAL VARIABLES IN THE MUNICIPALITY OF SÃO PAULO, SP. This study investigated the association of meteorological elements (minimum relative air humidity and daily maximum and minimum temperatures) with hospitalizations for respiratory morbidities (pneumonia and influenza) based on hospital reports for all age groups of the population of the city of Sao Paulo using the statistical technique of linear regression. The incidence of daily hospitalization of people with respiratory problems from the Unified Health System (SUS), specifically for influenza and pneumonia, was correlated with daily meteorological data from the meteorological station of the Institute of Astronomy and Geophysics of the University of São Paulo/USP from January 2002 to December 2005. From the annual charts of the entire study period, we observed a certain trend towards increased hospitalizations when maximum and minimum temperatures increased and the minimum relative humidity of the air decreased up to 7 days prior to admission peaks. However, results from linear regression tests indicated a low correlation between the number of hospitalizations for influenza and pneumonia for almost the entire period. Only between July and September 2004 were moderate correlations observed, considering that $47 \%$ of hospitalizations for influenza and pneumonia were associated with an increase in minimum temperatures and $35 \%$ of the maximum temperatures, coinciding with the driest periods for the year 2004. Between 2002 and 2005, the most vulnerable groups in the populations were children up to 4 years of age and the elderly 60 years and older.

Keywords: Influenza and pneumonia morbidity; Urban climatology; Municipality of São Paulo. 


\section{INTRODUÇÃO}

Os impactos da variabilidade climática na saúde humana são tratados a partir de aspectos epidemiológicos, considerando doenças infectocontagiosas como a malária, a dengue e a febre-amarela, cuja proliferação é dependente do regime de chuvas e do aumento da temperatura. CONFALONIERE (2003) trabalha com um modelo conceitual de vulnerabilidade social que considera os efeitos da variabilidade climática em relação a fenômenos extremos típicos do meio urbano como as tempestades e as enchentes nas grandes metrópoles. A relação entre meio ambiente e saúde, no que concerne aos fatores de risco à saúde humana (exposição a poluentes atmosféricos como monóxido e dióxido de carbono, dióxido de enxofre, material particulado etc.) tem sido estudada, principalmente, na dimensão temporal por meio de modelos estatísticos. Associações entre qualidade do ar e mortalidade por doenças respiratórias (BRAGA \& SALDIVA 2001, 2002, 2004) são exemplos de estudos realizados para o município de São Paulo no campo da saúde ambiental, que procuraram avaliar o impacto de condições adversas à saúde desde curtos intervalos de tempo (dias) a maiores períodos (meses e anos).

No centro das grandes cidades, onde se concentram maior número de prédios, fluxo de automóveis, indústrias e circulação de pessoas, as temperaturas tendem a ser mais elevadas do que nas áreas verdes e de baixa densidade de edificações e de pessoas, configurando as denominadas ilhas de calor.

Para LOMBARDO (1985) a ilha de calor urbana é uma característica universal do clima urbano, propiciando temperaturas mais elevadas nas zonas centrais da cidade em comparação com as zonas periféricas ou rurais. As variações térmicas nas zonas urbanas e rurais podem chegar até $10^{\circ} \mathrm{C}$ na cidade de São Paulo e ocorrem basicamente devido às diferenças de irradiação de calor entre as regiões edificadas e a massa vegetal.

MENDONÇA (2000) desenvolveu um estudo para o município de Curitiba, onde relaciona as variáveis do clima urbano e a repercussão para a saúde humana. Seu estudo constitui uma contribuição ao planejamento urbano, conduzindo a uma melhoria na qualidade de vida da população. No entanto, o autor ressalta que o clima urbano constitui apenas uma das múltiplas facetas da qualidade ambiental.

O trabalho aqui proposto foi o de desenvolver um estudo na área da Biometeorologia, subárea de pesquisa da Geografia Médica. Conforme MEADE et al. (p. 83, 1988):

“ a Biometeorologia se preocupa em esclarecer como as variações e mudanças nas caracte- rísticas físicas e químicas da atmosfera afetam variações e mudanças nos sistemas físico-químicos dos organismos dos seres humanos, bastante sensíveis 'as alterações atmosféricas de diversas ordens: temperatura, umidade, ventos, radiação solar, poluição atmosférica, pressão e descarga elétrica, magnetismo, etc.”

O tempo meteorológico consiste nas condições atmosféricas instantâneas, vivenciadas cotidianamente pelos seres humanos, sendo que suas interferências se tornam imediatas sobre as atividades da população. Ele se modifica em curtos intervalos no tempo e no espaço, de forma que seu comportamento dinâmico influencia nos aspectos biometeorológicos dos indivíduos. Diferentemente, o clima pode ser definido como o ambiente atmosférico constituído pela série de estados da atmosfera em sua totalidade (estados atmosféricos) sobre um lugar em sua sucessão habitual, englobando também os valores extremos que são mascarados pelas médias (STEINKE 2012).

Sob a óptica da Epidemiologia, explica-se a ocorrência de morbidades a partir de três pilares: i) qual a população de risco em adquiri-las, ii) qual a sua dimensão temporal (período de ocorrência mais comum em relação a dias, meses, estação sazonal e ano) e iii) a necessidade de se saber quais são as condições ambientais, sociais e culturais dos lugares onde elas mais ocorrem (PICKENHAYN 2009).

A gripe, causada pelo vírus influenza (Myxovirus influenzae) é uma das doenças respiratórias que mais acometem o homem. Essa doença possui a capacidade de mudar constantemente suas características, devido a mutações; desse modo, um mesmo indivíduo pode ter vários episódios de gripe ao longo de sua vida. Além disso, dissemina-se rapidamente, gerando epidemias e pandemias. Por esses motivos, é considerada uma doença potencialmente grave, que mata milhares de pessoas todos os anos (MINISTÉRIO DA SAÚDE 2005).

Já a pneumonia é uma doença que afeta cerca de 2,1 milhões de brasileiros todos os anos. Trata-se da principal causa de internação hospitalar e a quinta causa de morte no Brasil. Diferentemente da gripe, a pneumonia é causada por bactérias, vírus e fungos, sendo a bacteriana a mais comum (MINISTÉRIO DA SAÚDE 2005). Seus sintomas geralmente aparecem de forma aguda ou rápida, mas podem se desenvolver também lentamente; no início pode ser confundida com uma gripe ou resfriado forte. Em geral, ela afeta mais os idosos, pessoas com doenças crônicas ou com imunidade baixa, embora possa afetar também crianças, jovens e adultos saudáveis. 
Em crianças, a doença é a principal causa de morte em todo o mundo (BANCO DE SAÚDE 2008).

Dessa forma, o presente artigo objetiva investigar a associação dos elementos meteorológicos (umidade relativa mínima e temperaturas máximas e mínimas diárias) com as internações por pneumonia e gripe, registradas por meio das notificações hospitalares para todas as faixas-etárias da população paulistana para os anos de 2002 a 2005, utilizando técnica estatística de regressão linear, sem considerar a influência de poluentes. Além disso, pretendeu-se verificar qual parcela da população seria mais vulnerável segundo os aspectos demográficos, como sexo e faixa-etária.

\section{2 ÁREA DE ESTUDO}

São Paulo é a maior cidade e o mais importante centro econômico do Brasil. Conta com uma área de $1.509 \mathrm{~km}^{2}$ (PREFEITURA MUNICIPAL DE SÃO PAULO 2002), onde se distribui uma população de 10.927.985 habitantes, com uma concentração média de 7.077,4 habitantes $/ \mathrm{km}^{2}$, segundo estimativas do Instituto Brasileiro de Geografia e Estatística (IBGE 2000). As coordenadas geográficas do município são $46^{\circ} 38^{\prime} 10^{\prime \prime}$ de longitude oeste e $23^{\circ} 32^{\prime} 51^{\prime \prime}$ de latitude sul, o que lhe confere uma posição a sudeste do Estado (PREFEITURA MUNICIPAL DE SÃO PAULO 2002) (Figura 1).

A cidade de São Paulo situa-se em um alto platô, com uma altitude média de $750 \mathrm{~m}$ acima do nível do mar, e tem o clima classificado como tropical úmido de altitude (TARIFA \& ARMANI 2001). A altitude explica o fato das manhãs e noites serem frequentemente mais frescas. As quatro estações do ano não são claramente definidas. As temperaturas médias anuais variam entre $20{ }^{\circ} \mathrm{C}$ e $24^{\circ} \mathrm{C}$, podendo atingir $18^{\circ} \mathrm{C}$ ou menos nas regiões mais elevadas, devido aos efeitos conjugados da latitude, altitude e frequência das massas polares (TARIFA \& AZEVEDO 2001).

Durante o verão, principalmente em janeiro, é comum uma variação de temperatura máxima média entre $30{ }^{\circ} \mathrm{C}$ e $32{ }^{\circ} \mathrm{C}$, enquanto que nos meses de inverno, a média mínima de temperatura pode variar entre $6{ }^{\circ} \mathrm{C}$ e $20^{\circ} \mathrm{C}$, com mínimos absolutos variando de $4{ }^{\circ} \mathrm{C}$ a $8^{\circ} \mathrm{C}$. A média anual de precipitações é superior a $1.500 \mathrm{~mm}$. O período menos chuvoso ocorre no inverno (junho, julho e agosto), enquanto os períodos de chuva iniciam em dezembro e estendem-se até março (TARIFA \& REZENDE 2001).

De acordo com revisão sistemática de Clima e Saúde desenvolvida por RIBEIRO et al. (2016) a cidade de São Paulo já apresenta alteração na série histórica de precipitação e temperatura. Segundo relatório da estação meteorológica do IAG/USP (2010), a quantidade de dias sem chuva está aumentando. O padrão de chuva na cidade está se alterando, sugerindo influência da mudança climática global no regime de chuvas. Desse modo, pode-se inferir que grandes cidades recebem influências das mudanças climáticas e microclimáticas (RIBEIRO et al. 2016).

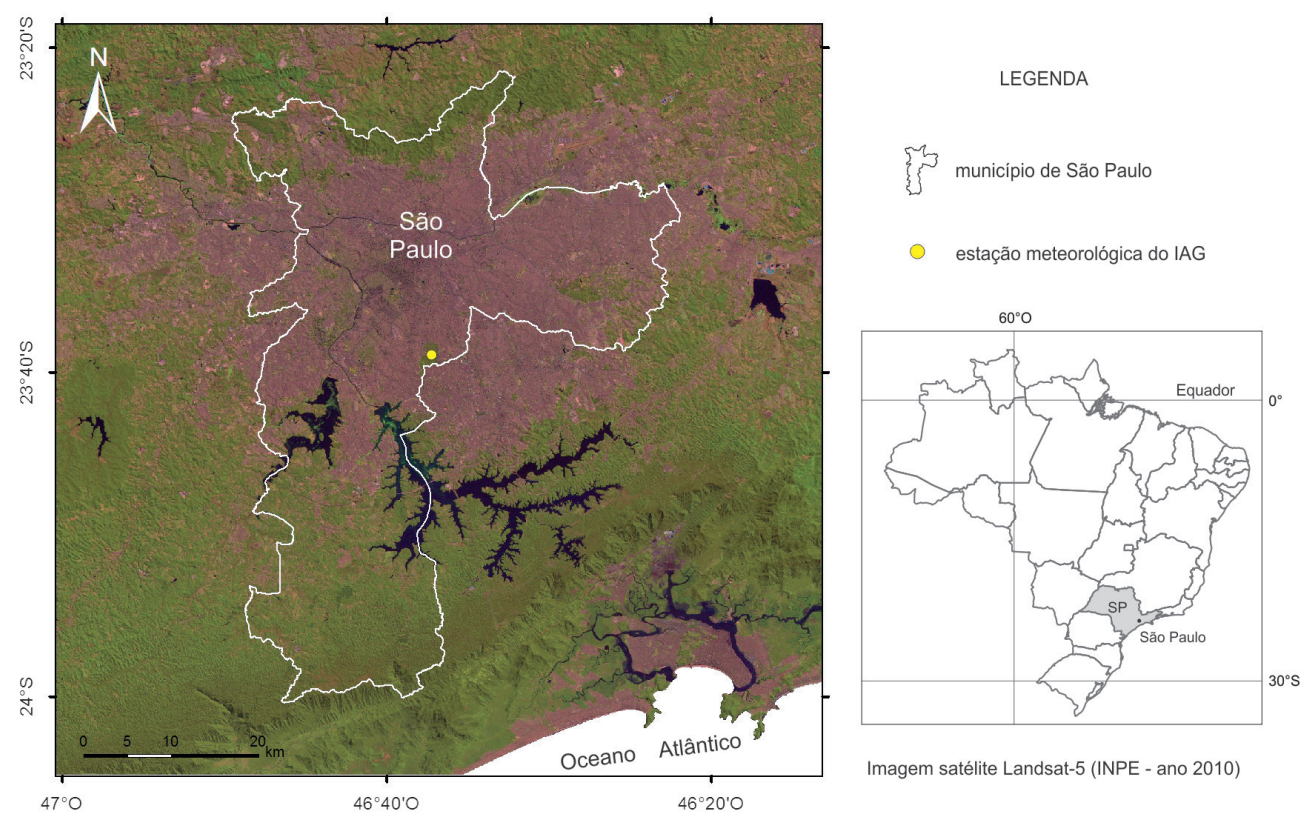

FIGURA 1 - Localização da cidade de São Paulo no estado de São Paulo e no Brasil e da estação meteorológica no MSP. 


\section{MATERIAL E MÉTODOS}

\subsection{Dados meteorológicos}

Foram utilizados dados diários de temperaturas máxima e mínima e umidade relativa mínima, elementos considerados fundamentais no condicionamento das pessoas à maior sensibilidade à infecção pela gripe ou pela pneumonia (CARNESECA et al. 2010, NATALI et al. 2011).

De modo geral, a umidade do ar é um dos fatores meteorológicos importantes para o conforto ambiental dos seres humanos, podendo causar sérios distúrbios à saúde, quando apresenta valores muito baixos persistentes por alguns dias. A umidade do ar é mais baixa principalmente no final do inverno e início da primavera, no período da tarde, entre 14 e $16 \mathrm{~h}$. A umidade se eleva sempre que chove, devido à evaporação que ocorre posteriormente em áreas florestadas ou próximas aos rios ou represa, ou quando a temperatura diminui (orvalho) (CEPAGRI/UNICAMP 2007).

Alguns problemas decorrentes da baixa umidade do ar podem ser registrados, tais como: complicações alérgicas e respiratórias devido ao ressecamento de mucosas, sangramento pelo nariz, ressecamento da pele, irritação dos olhos, eletricidade estática nas pessoas e em equipamentos eletrônicos e aumento do potencial de incêndios em pastagens e florestas (CEPAGRI/UNICAMP 2007).

Os dados meteorológicos diários foram obtidos da estação meteorológica do Instituto de Astronomia, Geofísica e Ciências Atmosféricas da Universidade de São Paulo (IAG/USP) (Figura 1), entre janeiro de 2002 a dezembro de 2005.

\subsection{Dados de morbidades}

Inicialmente foi feita uma análise dos dados referentes à rede de admissões diárias por hospitais atendidos pelo SUS no município de São Paulo no período de janeiro de 2002 a dezembro de 2005, com autorização do Comitê de Ética em Pesquisa - CEP da Secretaria Municipal de Saúde. Para o estudo, foram analisadas apenas as internações de pacientes com residência no município de São Paulo.

Foram extraídos os casos de internação diária associadas ao sub-grupo Influenza e Pneumonia (J10 a J18) - Código Internacional de Doenças (CID - 10) - por sexo e faixa etária, através do software estatístico Epi Info, totalizando 91.124 internações.
3.3 Associação entre as variáveis meteorológicas e de saúde

Testes de regressão linear foram realizados a fim de avaliar o grau de associação entre os dados meteorológicos diários (Temperatura Mínima Diária - Tmin, Temperatura Máxima Diária - Tmax e Umidade Relativa Mínima Diária - URmin) e de saúde (número de internações hospitalares diárias por influenza e pneumonia).

Baseado no recorte temporal adotado por RIBEIRO (1996), os testes de regressão linear foram feitos a cada 90 dias para se constatar quais os meses e anos em que os elementos meteorológicos demonstrariam maior significância e coeficiente de determinação mais elevado.

\subsection{Cálculo das taxas de internação}

A partir da base municipal Tabnet/DATASUS da Secretaria Municipal de Saúde de São Paulo, foram coletados os dados da população total do município de São Paulo, população de idosos (acima de 60 anos) e de crianças (abaixo de 4 anos), para o cálculo das taxas de internação de gripe e pneumonia da Rede SUS.

A taxa de internação da população total foi calculada para os meses de janeiro a dezembro durante os anos de 2002 a 2005 (considerando que a população total não variou de um mês para outro), segundo a equação:

\section{Total de Internados \\ População Total}

De acordo com o DATASUS, a população total foi fixada em 10.600.059 pessoas para o ano de 2002, 10.677.017 em 2003, 10.753.768 em 2004 e 10.927.985 em 2005.

\section{RESULTADOS}

As tabelas 1, 2 e 3 ilustram os valores obtidos por meio dos testes de regressão linear. Somente no intervalo de julho a setembro de 2004 obteve-se valores de coeficiente de determinação mais elevados, coincidindo com o período tipicamente mais seco do ano: 0,417 (Figura 2) para a relação entre Temperatura Mínima Diária e Internação por Gripe e Pneumonia e 0,3478 (Figura 3) para a relação entre Temperatura Máxima Diária e Internação por Gripe e Pneumonia. 
TABELA 1 - Temperatura Mínima Diária (IAG/USP) versus Internação por gripe e pneumonia (Rede SUS).

\begin{tabular}{lccrr}
\cline { 2 - 4 } & \multicolumn{4}{c}{ Coeficiente de determinação $\left(r^{2}\right)$ : } \\
\cline { 2 - 5 } & 2002 & 2003 & 2004 & 2005 \\
\hline Janeiro a Março & 0,0961 & 0,0659 & 0,1053 & 0,0057 \\
\hline Abril a Junho & 0,0028 & 0,0938 & 0,1637 & 0,1412 \\
\hline Julho a Setembro & 0,0523 & 0,0072 & 0,4717 & 0,0095 \\
\hline Outubro a Dezembro & 0,005 & 0,0243 & 0,0008 & 0,0007 \\
\hline
\end{tabular}

TABELA 2 - Temperatura Máxima Diária (IAG/USP) versus Internação por gripe e pneumonia (Rede SUS).

\begin{tabular}{lrrrr}
\cline { 2 - 4 } & \multicolumn{3}{c}{ Coeficiente de determinação $\left(r^{2}\right)$ : } \\
\cline { 2 - 5 } & 2002 & 2003 & 2004 & 2005 \\
\hline Janeiro a Março & 0,1417 & 0,0008 & 0,002 & 0,0408 \\
\hline Abril a Junho & 0,0381 & 0,0009 & 0,1239 & 0,1716 \\
\hline Julho a Setembro & 0,0239 & 0,1022 & 0,3478 & 0,042 \\
\hline Outubro a Dezembro & 0,006 & 0,0016 & 0,0007 & 0,0236 \\
\hline
\end{tabular}

TABELA 3 - Umidade Mínima Diária (IAG/USP) versus Internação por gripe e pneumonia (Rede SUS).

\begin{tabular}{lrrrr}
\cline { 2 - 4 } & \multicolumn{4}{c}{ Coeficiente de determinação $\left(r^{2}\right)$ : } \\
\cline { 2 - 5 } & 2002 & 2003 & 2004 & 2005 \\
\hline Janeiro a Março & 0,105 & 0,0178 & 0,0151 & 0,0455 \\
\hline Abril a Junho & 0,1047 & 0,001 & 0,049 & 0,0456 \\
\hline Julho a Setembro & 0,048 & 0,119 & 0,059 & 0,1253 \\
\hline Outubro a Dezembro & 0,0115 & 0,019 & 0,0007 & 0,0133 \\
\hline
\end{tabular}

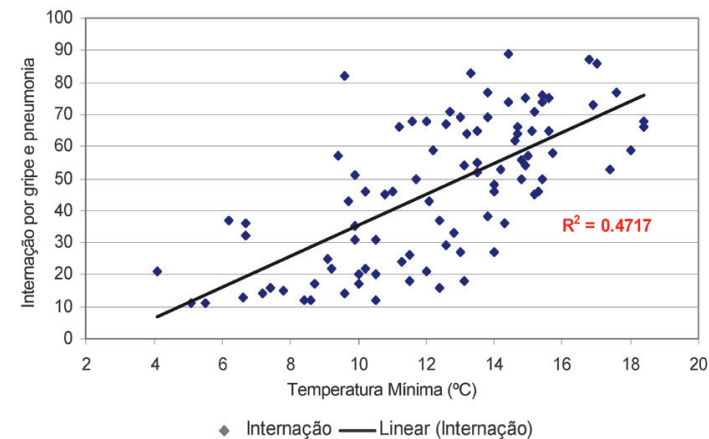

FIGURA 2 - Relação entre Temperatura Mínima Diária (IAG/USP) e Internação por gripe e pneumonia $-\mathrm{Jul} / \mathrm{Set} 2004$.

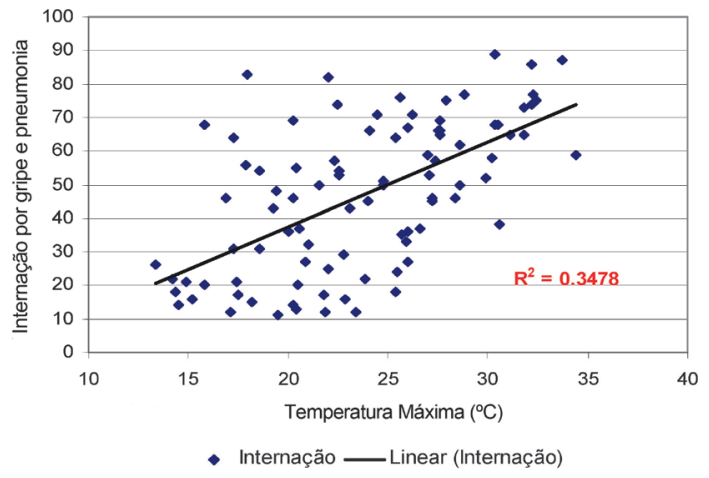

FIGURA 3 - Relação entre Temperatura Máxima Diária (IAG/USP) e Internação por gripe e pneumonia - Jul/Set 2004. 
Não se observou correlação estatística significativa com a Umidade Mínima Diária $(0,059$ - Figura 4), embora o ajuste da reta sugira uma relação inversamente proporcional entre os picos de internação por gripe e pneumonia e de Umidade Mínima Diária em grande parte dos meses de julho, agosto e setembro de 2004. Quando a umidade mínima diária é baixa (abaixo de 30\%), os picos de internação ultrapassam a 80 pacientes por dia, tendo ocorrido 3 dias após o ápice da queda da umidade (Figura 5).

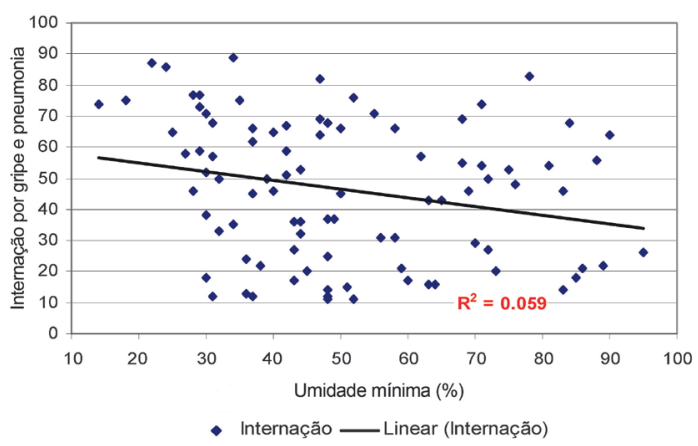

FIGURA 4 - Relação entre Umidade Mínima Diária (IAG/USP) e Internação por gripe e pneumonia - Jul/Set 2004.

Esperava-se que a Umidade Relativa Mínima apresentasse um coeficiente de determinação maior em relação aos picos de internação nos períodos mais secos do ano, fato não comprovado pelas regressões lineares, uma vez que o $\mathrm{r}^{2}$ para o período de julho a setembro de 2004 foi 0,059 (Figura 4).
Já para as temperaturas máximas e mínimas diárias a relação é diretamente proporcional (Figura 6), ou seja, para os três meses considerados, o número de internações aumenta com a elevação das temperaturas máxima e mínima, corroborado pelos maiores valores de $\mathrm{r} 2\left(\mathrm{r}^{2}=0,4717\right.$ e 0,3478 , respectivamente) (Figuras 2 e 3 ).

As internações por gripe e pneumonia foram analisadas por faixa-etária e sexo (Figura 7). Observa-se que as crianças menores de um ano e de um a quatro anos são as faixas de maior vulnerabilidade entre os jovens. Já as faixas etárias de 50 a 59 anos e acima de 60 anos são as com maior número de internações, sobretudo a última (quase $35 \%$ do total). O número de internações de indivíduos do sexo masculino é maior do que do sexo feminino, exceto na faixa acima de 60 anos, devido à menor expectativa de vida de indivíduos do sexo masculino. Para os indivíduos do sexo feminino a expectativa de vida é de 76,1 anos, enquanto que a do sexo masculino é de 72,3 anos (IBGE 2000).

Nota-se que as crianças de 0 a 4 anos e de 1 a 4 são internadas com muito mais frequência, assim como os idosos acima de 60 anos, correspondendo às faixas etárias de maior vulnerabilidade em relação a gripe e pneumonia (Figura 7).

A distribuição das taxas de internação por gripe ou pneumonia ao longo dos meses do ano de 2002 é menor quando comparadas aos anos de 2003, 2004 e 2005 (Figura 8). A menor taxa registrada $(7,6) \mathrm{em}$ 2002 foi em fevereiro, correspondendo a 7-8 pessoas internadas para cada grupo de 100.000 habitantes, enquanto a maior foi no mês de abril $(19,71)$.

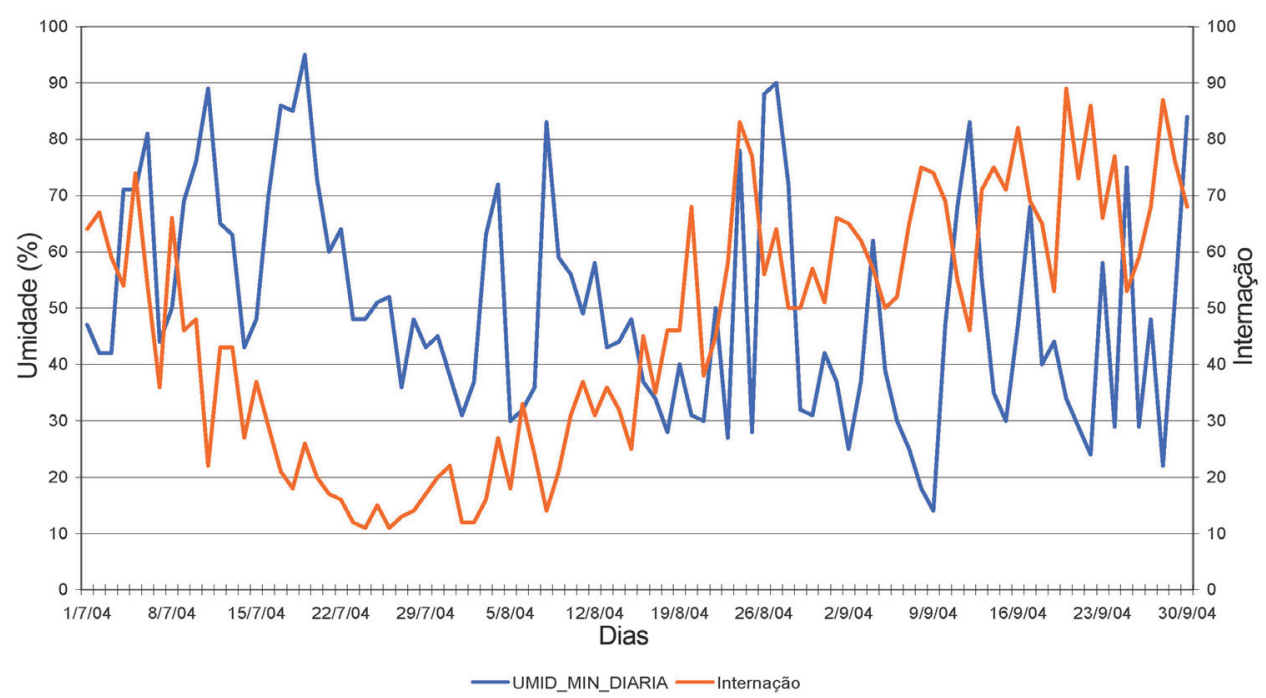

FIGURA 5 - Internação por gripe e pneumonia e a variabilidade da Umidade Mínima Diária Estação IAG/USP - Jul/Set 2004. 


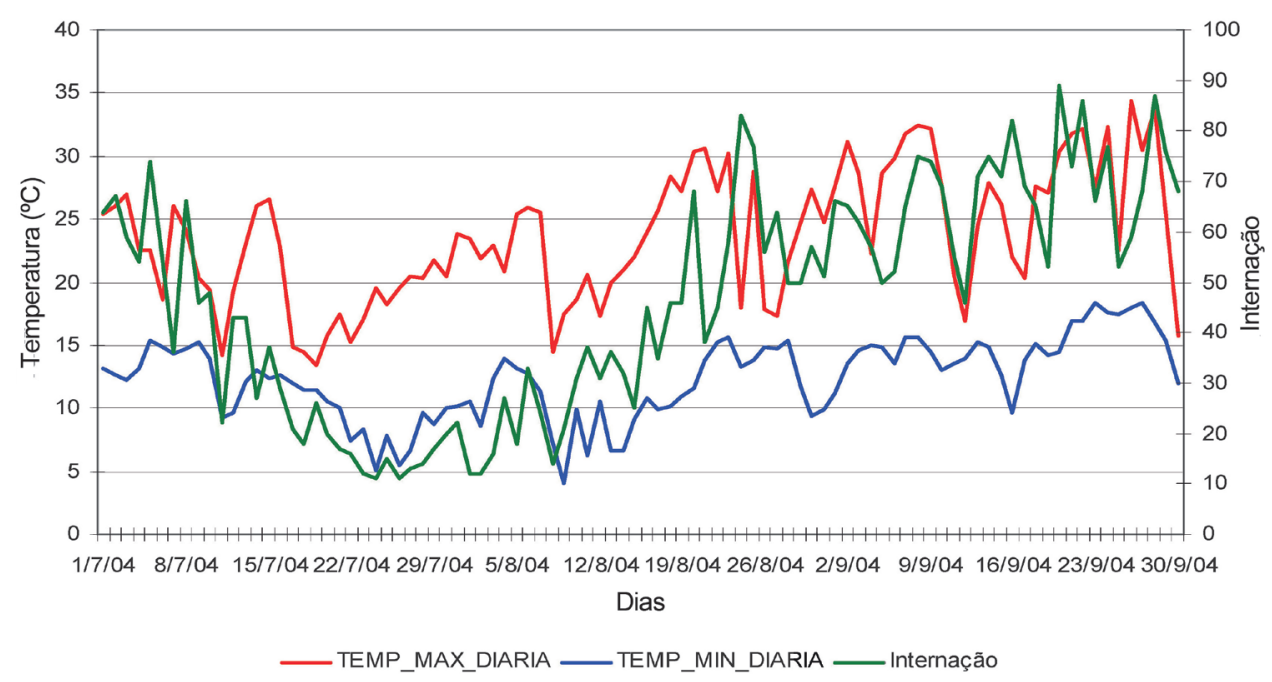

FIGURA 6 - Relação entre as Temperaturas Máximas e Mínimas Diárias da estação do IAG/ USP com internação por gripe e pneumonia entre os meses de julho a setembro de 2004.

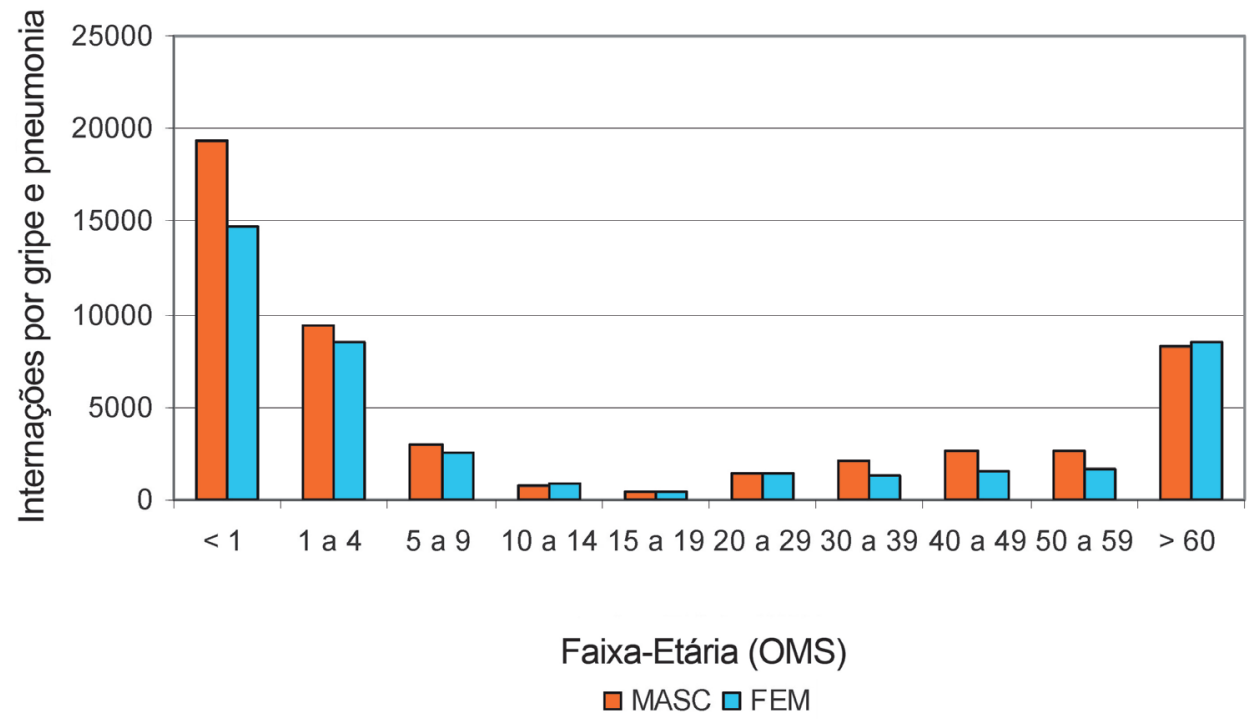

FIGURA 7 - Total de Internações por Influenza e Pneumonia durante os anos de 2002 a 2005 da Rede SUS na cidade de São Paulo, separados por sexo e faixa-etária.

Os picos ocorrem nos meses de abril e maio e depois em outubro, coincidindo com a transição da estação sazonal do verão no final de março e entrada do outono no final de setembro. Porém as taxas se mantêm altas nos meses de junho, julho e agosto, durante o inverno, estação sazonal comumente seca e com temperaturas máximas e mínimas mais baixas.

Depois de um período seco, com a entrada da primavera no mês de setembro, costuma-se ter o registro das temperaturas mais altas e mais baixas do ano, ou seja, períodos de maior amplitude térmica, sendo o organismo mais vulnerável a esse cho- que térmico ou às transições de estação sazonal, o que ajuda a explicar o pico de internação por gripe e pneumonia do mês de outubro.

Durante o ano de 2003, verificou-se que a menor taxa registrada foi em janeiro $(12,9)$ e a maior em abril $(29,47)$. Em 2004 a menor taxa registrada foi em julho $(9,5)$ e a maior em abril $(26,89)$. Esse ano mostrou um comportamento atípico, por ter apresentado a menor taxa no ápice do inverno, estação sazonal fria e seca. Em 2005 a menor taxa registrada foi de 12,67 no mês de janeiro e a maior no mês de abril $(28,29)$ (Figura 8 ). 

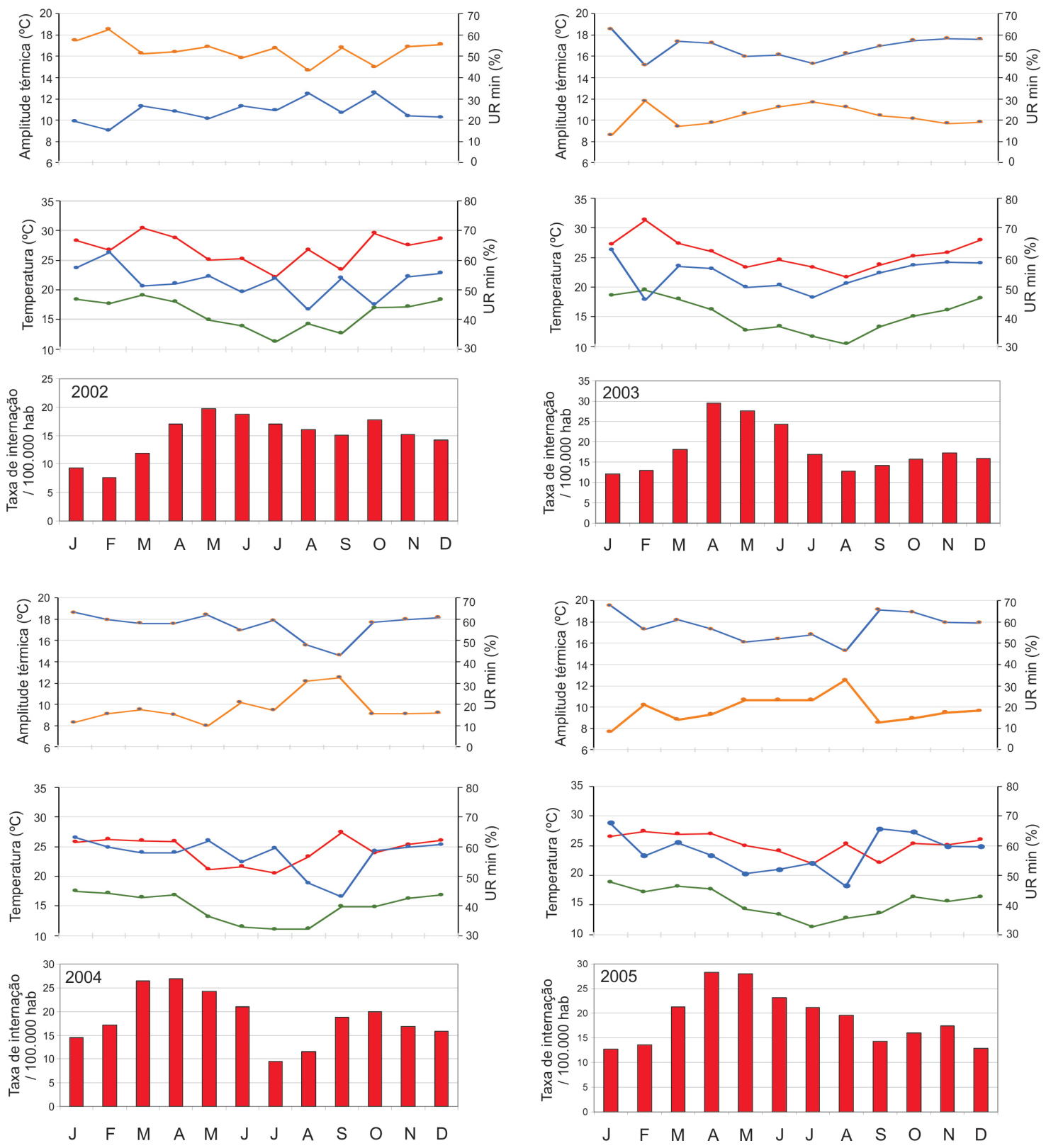

$\rightarrow T \max \rightarrow T \min \rightarrow$ UR mim $\rightarrow$ Amplitude térmica

FIGURA 8 - Taxa de internação por gripe e pneumonia no município de São Paulo para os anos de 2002 a 2005 e distribuição mensal de temperatura máxima mensal (Tmax), temperatura mínima mensal (Tmin) e umidade relativa mínima (UR min) e amplitude térmica mensal.

\section{DISCUSSÃO}

Os resultados dos testes de regressão linear, feitos a cada 90 dias, indicaram uma baixa correlação entre o número de hospitalizações por influenza e pneumonia e as variáveis meteorológicas para quase todo o período. Os testes apresentaram maior significância e um coeficiente de determinação mais elevado somente no intervalo de julho a setembro de 2004. Observou-se que $47 \%$ das internações por gripe e pneumonia estão associadas à ocorrência de baixas temperaturas durante as madrugadas e $35 \%$ são decorrentes das temperaturas máximas observadas durante as tardes, nos períodos mais secos do ano. Nesses casos, as internações nesse período são reflexos da umidade do ar abaixo de $30 \%$. Os picos de internação ultrapassam 80 pacientes por dia, após 
um período anterior de aproximadamente três dias de baixa umidade.

Nesse mesmo período, observa-se que para as temperaturas máximas e mínimas diárias a relação é diretamente proporcional aos números de internação, causada em cerca de $47 \%$ dos casos, devido ao aumento das temperaturas máxima e mínima.

Comparando-se a variação diária das taxas de internação com os parâmetros climáticos, observou-se que pode haver um atraso de até 7 dias dos picos de internação de gripe e pneumonia em relação aos picos de aumento das temperaturas máxima e mínima (Figura 6) e queda da umidade relativa mínima (Figura 5). Isso pode ser explicado pelo fato das respostas biometeorológicas das alterações de tempo não serem sentidas imediatamente pela população exposta (WANG et al. 2003).

Em artigo sobre temporalidade realizado em período semelhante ao do presente estudo, CARNESECA et al. (2010) também correlacionaram a contagem diária de hospitalizações por pneumonia e por doenças crônicas na cidade de São Paulo com as covariáveis temperatura, umidade relativa do ar, estações do ano e dias da semana, utilizando o Modelo de Regressão de Poisson. Os autores verificaram que tanto o número de internações por pneumonia, quanto por doenças crônicas, foram influenciados pela umidade do ar e pela temperatura, especialmente nas faixas etárias de crianças e idosos.

Semelhante ao observado nesse estudo, NATALI et al. (2011) identificaram em estudo ecológico de séries temporais de internações hospitalares por doenças respiratórias em hospitais conveniados ao SUS na cidade de São Paulo, entre 2000 e 2004, uma tendência de aumento das internações ao longo do período analisado, além da constatação do pico de morbidade no começo do outono. Observou-se também que as internações de crianças e adolescentes apresentaram padrão de distribuição dependente da faixa etária e da sazonalidade: quanto menor a faixa etária maior o número de internações.

Uma limitação dos dados provenientes da Autorização de Internação Hospitalar (AIH) do Sistema Único de Saúde é que eles não representam o universo de internações do município de São Paulo, abrangendo possivelmente cerca de $40 \%$ do número total de internações realizadas durante o período em estudo. Como essa cobertura se refere ao contingente populacional mais carente, compreende-se que os resultados dessa análise abrangem um grupo específico da população paulistana.
Outra limitação dessa base de dados secundária consiste na sua confiabilidade de registro dos prognósticos, sujeitos a algumas discrepâncias relacionadas ao perfil epidemiológico, já que os prontuários podem apresentar erros de codificação que não reproduzem com acurácia o motivo de internação.

\section{CONSIDERAÇÕES FINAIS}

O objetivo principal do estudo foi identificar a influência de variáveis meteorológicas nos eventos de morbidade de gripe e de pneumonia e suas ocorrências nas faixas etárias mais vulneráveis no município de São Paulo.

Os resultados indicaram tendência do aumento das internações quando há aumento das temperaturas máximas e mínimas, e queda da umidade relativa mínima em até 7 dias antes dos picos de internação. No entanto, os resultados dos testes de regressão linear indicaram uma baixa correlação entre o número de hospitalizações por influenza e pneumonia e variáveis meteorológicas para quase todo o período. Apenas entre os meses de julho e setembro de 2004 que apresentaram correlações médias, tendo em vista que $47 \%$ das internações por influenza e pneumonia estavam associadas ao aumento das temperaturas mínimas e 35\% das temperaturas máximas, coincidentes com os períodos mais secos do ano de 2004. Entre 2002 e 2005, destaca-se que os grupos de populações mais vulneráveis são as crianças de até 4 anos de idade e os idosos a partir de 60 anos que apresentaram as maiores taxas de incidência.

Os resultados indicaram que as maiores incidências de internações ocorrem em crianças do sexo masculino menores de 1 ano e em idosos maiores do que 60 anos do sexo feminino, principalmente nos meses de abril e maio para os anos de 2002 a 2005.

A relação entre clima e saúde, por si só, não é suficiente para explicar os problemas de saúde relacionados aos diagnósticos de influenza e pneumonia, mas pode fornecer valiosas ferramentas para orientação de programas e demandas de serviço de vigilância em saúde ambiental e epidemiológica.

\section{REFERÊNCIAS BIBLIOGRÁFICAS}

BANCO DE SAÚDE. 2008. CID 10 Classificação Internacional de Doenças e Problemas Relacionados à Saúde. Disponível em http:// www.bancodesaude.com.br. Acessado em 29 mar. 2008. 
BRAGA, L.F.A.; SALDIVA, P.H.N. 2001. Relação entre poluição atmosférica e atendimentos por infecção de vias aéreas superiores no município de São Paulo: avaliação do rodízio de veículos. Revista Brasileira de Epidemiologia, 4(3): 220-229.

BRAGA, L.F.A.; SALDIVA, P.H.N. 2002. Poluição atmosférica e atendimentos por pneumonia e gripe em São Paulo, Brasil. Revista de Saúde Pública, 36(1): 88-94.

BRAGA, L.F.A.; SALDIVA, P.H.N. 2004. Internações e óbitos e sua relação com a poluição atmosférica em São Paulo, 1993 a 1997. Revista de Saúde Pública, 38(6): 751-757.

CARNESECA, E.C.; ACHCAR, J.A.; MARTINEZ, E.Z.; ALONSO, J.B.; HOTTA, L.K. 2010. Contagem diária de hospitalizações e variações climáticas na cidade de São Paulo: uma abordagem bayesiana. Revista Brasileira de Biometeorologia, 28(1): 57-72.

CEPAGRI/UNICAMP - CENTRO DE PESQUISAS METEOROLÓGICAS E CLIMÁTICAS APLICADAS À AGRICULTURA / UNIVERSIDADE ESTADUAL DE CAMPINAS. 2007. Umidade do ar e saúde no inverno: cuidados a serem tomados. Escala psicrométrica Unicamp para indicação de niveis de umidade relativa do ar prejudiciais à saúde humana. CEPAGRI, UNICAMP, Campinas. Dísponível em http://www.cpa. unicamp.br/artigos-especiais/umidade-doar-saude-no-inverno.html. Acessado em 21 set. 2007.

CONFALONIERI, U.E.C. 2003. Variabilidade climática, vulnerabilidade social e saúde no Brasil. Revista Terra Livre, 19(I): 193-204.

IAG/USP - INSTITUTO DE ASTRONOMIA, GEOFÍSICA E CIÊNCIAS ATMOSFÉRICAS/ UNIVERSIDADE DE SÃO PAULO. 2010. São Paulo: IAG/USP/Seção Técnica do Serviço Meteorológico (Boletim Climatológico Anual da Estação Meteorológica do IAG, 14).

IBGE - INSTITUTO BRASILEIRO DE GEOGRAFIA E ESTATÍSTICA. 2000. Censo Demográfico 2000. Disponível em http// www.ibge.gov.br. Acessado em 23 mar. 2008.
LOMBARDO, M.A. 1985. Ilha de calor nas metrópoles: o exemplo de São Paulo. Hucitec, São Paulo, 244 p.

MEADE, F.; FLORIN, J.; GESLER, W. 1988. Medical Geography. The Guilford Press, New York, 340 p.

MENDONÇA, F. 2000. O clima urbano de cidades de porte médio e pequeno: aspectos teóricos - metodológico e estudo de caso. In: J.L. Sant'Anna Neto, J.A. Zavatini (Org.) Variabilidade e mudanças climáticas: implicações ambientais e socioeconômicas. Maringá, Eduem, p.167-192.

MINISTÉRIO DA SAÚDE. 2005. Guia de Vigilância Epidemiológica. Brasília, $6^{\mathrm{a}}$ edição, 815 p.

NATALI, R.M.T; SANTOS, D.S.P.S.; FONSECA, A.M.C.; FILOMENO, G.C.M.; FIGUEIREDO, A.H.A.; TERRIVEL, P.M.; MASSONI, K. M.; BRAGA, A.L.F. 2011. Perfil de internações hospitalares por doenças respiratórias em crianças e adolescentes da cidade de São Paulo, 2000-2004. Revista Paulista de Pediatria, 29(4): 584-590.

PICKENHAYN, J. 2009. Salud y enfermedad en geografía. Lugar Editorial, Buenos Aires, 156 p.

PREFEITURA MUNICIPAL DE SÃO PAULO. 2002. Atlas Ambiental do Munício de São Paulo. H. Takiya (org.). Prefeitura Municipal de São Paulo/SVMA-SEMPLA, São Paulo. Disponível em: http://atlasambiental.prefeitura.sp.gov.br. Acessado em 12 fev. 2008.

RIBEIRO, H. 1996. Ilha de calor na cidade de São Paulo: sua dinâmica e efeitos na saúde da população. Faculdade de Saúde Pública, Universidade de São Paulo, Tese de Livre Docência, 144 p.

RIBEIRO, H.; PESQUERO, C.R.; COELHO, M.S.Z.S. 2016. Clima urbano e saúde: uma revisão sistematizada da literatura recente. Estudos Avançados, 30(86): 67-82.

STEINKE, E.T. 2012. Climatologia Fácil. Oficina de Textos, São Paulo, 144 p.

TARIFA, J.R.; ARMANI, G. 2001. Os climas 'naturais'. In: J.R. Tarifa \& T.R. Azevedo (orgs.) Os climas na cidade de São Paulo: teoria e 
prática. São Paulo, GEOUSP (Coleção Novos Caminhos, 4), p. 34-46.

TARIFA, J.R.; AZEVEDO, T.R. 2001. Os climas na cidade de São Paulo: teoria e prática. São Paulo, GEOUSP, 199 p. (Coleção Novos Caminhos, 4).
WANG, Y; LEVI, C.R; ATTIA, J.R; D’ESTE, C.A; SPRATT, N.; FISHER, J. 2003. Seasonal variation in stroke in the Hunter Region, Australia: A 5-Year Hospital-Based Study, 1995 - 2000. Stroke, 34: 1144-1150.

\section{Endereço do autor:}

Marina Jorge de Miranda - Coordenadoria de Vigilância em Saúde Ambiental (CGVAM), Secretaria de Vigilância em Saúde (SVS), Ministério da Saúde, prédio PO 700, $6^{\circ}$ andar, Asa Norte, CEP 70719040, Brasília, DF, Brasil.E-mail: marina.miranda@saude.gov.br

Artigo submetido em 9 de dezembro de 2016, aceito em 25 de janeiro de 2017. 